\title{
Inhibition of return: Unraveling a paradox
}

\author{
ELINA BIRMINGHAM \\ University of British Columbia, Vancouver, British Columbia, Canada \\ Troy A. W. Visser AND JANice J. SNYder \\ University of British Columbia, Okanagan, British Columbia, Canada \\ AND \\ Alan Kingstone \\ University of British Columbia, Vancouver, British Columbia, Canada
}

\begin{abstract}
Although inhibition of return (IOR) is widely believed to aid search by discouraging reexamination of previously inspected locations, its impact actually appears to decline as the number of target locations increases. We test three possible reasons for this paradoxical result: (1) IOR is capacity-limited, (2) IOR is sensitive to subtle changes in target location probability, and (3) IOR decays with distance from a previously attended location. The present investigation provides strong support for the third explanation, indicating that a gradient of inhibition is centered on previously attended locations. We note that this inhibitory gradient resolves a paradox in the literature. Moreover, we speculate that the inhibitory gradient may reflect a "similarity space" within which target locations near to the cue are tagged with inhibition due to their similarity to the cued location. The farther the target location is away, the less similar it is to the cued location, and thus the less inhibition it receives.
\end{abstract}

Over 20 years ago, Posner and Cohen (1984) reported that when an abrupt peripheral onset (cue) precedes the appearance of a visual target, responses are faster for targets at a cued location at relatively short cue-target onset asynchronies (CTOAs), but slower at relatively longer CTOAs (e.g., $300 \mathrm{msec}$ or more). This slowing of response times (RTs) has been termed inhibition of return (IOR; Posner, Rafal, Choate, \& Vaughan, 1985).

Whereas facilitation has conventionally been attributed to the fact that the cue draws attention to the target location, thereby facilitating target processing, an explanation for IOR is more complex. It is thought that attention is first withdrawn from a cued location when a target is not detected there. Because this alone cannot explain why responses are slower at cued than uncued locations, however, it is additionally surmised that attention is actively inhibited from returning to a recently cued/attended location, thereby delaying target processing (see, e.g., Gibson \& Egeth, 1994; Maylor, 1985; Reuter-Lorenz, Jha, \& Rosenquist, 1996).

Inhibition of return is often thought to reflect the output of a mechanism that biases visual search toward novel locations, thereby improving search efficiency (Klein, 1988; Posner \& Cohen 1984). This link has been bolstered by evidence that IOR is larger when search is difficult (Klein \& MacInnes, 1999; Snyder \& Kingstone, in press), and that IOR can co-occur at multiple locations (see, e.g., Danziger, Kingstone, \& Snyder, 1998). Para- doxically, however, the overall IOR effect seems to decline as the number of target locations increases (Pratt, Adam, \& McAuliffe, 1998). This is surprising because it seems that in an environment with more target locations, it would be beneficial to increase inhibition and thus strengthen the bias toward examining novel locations.

The present work investigates three possible explanations for why IOR declines as the number of target locations increases. Determining which of these explanations is accurate has significant implications for understanding the mechanisms underlying IOR and visual search. One possibility is that capacity limitations are responsible for the decline in IOR as the number of locations increases (Castel, Pratt, \& Craik, 2003; Maylor, 1985). Pratt et al. (1998) describe this capacity-limited account as follows:

. . . if IOR does reflect the output of an attentional mechanism that improves searches, one might expect that the number of locations where the target might potentially occur would have a negative influence on the magnitude of IOR. Assuming that some amount of attention is allocated to each target location placeholder in a display, larger number of placeholders should require a greater proportion of the available attentional resources. This leads to a situation in which the amount of inhibition might be expected to decrease with increasing numbers of potential target locations because it would become more difficult to 
keep track of novel and previously attended locations. (p. 213)

In a nutshell, then, the capacity-limited account predicts that as the number of possible target locations increases, resources required for inhibition should be depleted. As a consequence, cued and uncued RTs should become more similar, effectively reducing IOR (i.e., the difference between cued and uncued RTs).

A second possibility is that changes in spatially nonpredictive target location probability, routinely confounded with changes in the number of possible target locations, produce top-down adjustments in IOR. For example, with a nonpredictive cue the probability of a target appearing at a cued location declines from .50 to .125 as the number of target locations increases from two to eight. Because of this, participants may choose to allocate less inhibition to cued locations when it is less likely that a target will appear in any one of them. This probability account is consistent with findings that IOR is mediated by strategic factors (see, e.g., Castel, Pratt, Chasteen, \& Scialfa, 2005; Lupiáñez, Milliken, Solano, Weaver, \& Tipper, 2001), and it is also consistent with the notion that IOR reflects the output of a flexible forage facilitator (e.g., Klein, 1988).

A third possibility is that increasing the number of target locations changes the baseline against which IOR is measured (namely, RTs at uncued locations), thereby yielding a decrease in mean IOR (see, e.g., Pratt et al., 1998). According to this gradient account, the strength of inhibition declines gradually with increasing distance from the cued location. Moreover, as the number of target locations increases, the proximity between cued and uncued locations increases as well. This is because potential target locations are usually spaced symmetrically about fixation, and thus the average distance between cued locations and target locations is necessarily confounded with set size. As a result, being located closer to the epicenter of inhibition, uncued target RTs will be slower, and overall IOR will decrease.

Importantly, extant results cannot be used to distinguish between the capacity-limited, probability, and gradient accounts because all previous studies have confounded changes in target location probability and number of target locations. Thus, all three accounts predict the same pattern of results - namely, a decline in IOR with an increase in number of target locations. What is needed is to manipulate target location probability and the number of locations independently. To this end, in the present study we began by running the standard (confounded) probability-location manipulation to replicate the baseline effect that has been established in the literature. Then, with different groups of participants, we manipulated either target location probability, holding number of locations constant, or number of locations, holding target location probability constant.

\section{Predictions}

The capacity-limited account predicts that IOR should be affected by changes in the number of locations whether or not this change is confounded with probability. The probability account predicts that IOR should be affected by changes in target location probability, whether or not this change is confounded with number of locations, but not by changes in number of locations alone. Note that in neither case are changes in proximity of potential target locations predicted to be important. On the other hand, the gradient account predicts that RTs will be slower at target locations near the cued location (yielding a smaller IOR effect) and, conversely, faster at target locations far from the cued location (yielding a larger IOR effect).

\section{METHOD}

\section{Participants}

One hundred and five undergraduates from the University of British Columbia (who received course credit) were assigned randomly to one of three conditions: 35 students ( 22 female, 13 male; mean age $=20.41$ years, age range $=17-27)$ in the standard condition (location number and probability confounded); 36 students (21 female, 15 male; mean age $=20.31$ years, age range $=17-37$ ) in the probability-only condition; and 34 students ( 26 female, 8 male; mean age $=21.15$ years, age range $=18-32$ ) in the location-only condition. All observers reported normal or corrected-to-normal vision.

\section{Apparatus and Stimuli}

Stimuli were presented on a 20 -in. monitor connected to a computer that presented the stimuli and recorded RTs in milliseconds. The display consisted of square gray placeholders (subtending $2^{\circ}$ ) on a black background, with either a central gray placeholder or cross as the fixation marker. In the standard and location-only conditions, the displays consisted of 2, 3, 4, or 6 peripheral placeholders, and in the probability-only condition, the displays consisted of two peripheral placeholders. Placeholders were equally spaced around an imaginary circle (radius $8.5^{\circ}$ ), with $0^{\circ}$ represented at 12 o'clock in Figure 1. In the two-location condition, two placeholder boxes were aligned opposite each other, at $0^{\circ}$ and $180^{\circ}$ (or at $90^{\circ}$ and $270^{\circ}$ ). In the three-location condition, three placeholders were located at the corners of a notional triangle, at $0^{\circ}, 120^{\circ}$, and $240^{\circ}$ (or at $180^{\circ}, 300^{\circ}$, and $\left.60^{\circ}\right)$. In the four-location condition, four placeholders were located at the ends of a notional plus sign, at $0^{\circ}, 90^{\circ}, 180^{\circ}$, and $270^{\circ}$ (or at $45^{\circ}, 135^{\circ}, 225^{\circ}$, and $315^{\circ}$ ). Finally, in the six-location condition, placeholders were located at the corners of a notional hexagon, at

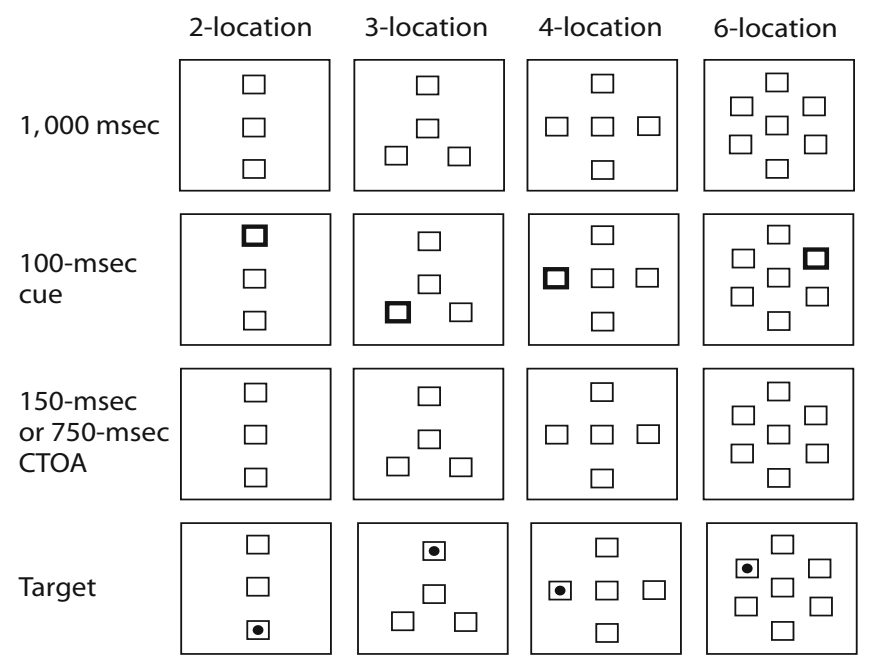

Figure 1. Trial sequence for the two-location, three-location, four-location, and six-location conditions of the standard and location-only conditions. 
$0^{\circ}, 60^{\circ}, 120^{\circ}, 180^{\circ}, 240^{\circ}$, and $300^{\circ}$ (or at $30^{\circ}, 90^{\circ}, 150^{\circ}, 210^{\circ}, 270^{\circ}$, and $330^{\circ}$ ). In order to control for location effects, half of participants received the configuration shown in Figure 1, and the other half received the same configuration but rotated (exact positions given in parentheses above). The cue was a brightening of one of the placeholders. The target was a gray asterisk measuring $0.5^{\circ}$ appearing at the center of a placeholder.

\section{Procedure}

Conditions were divided into four counterbalanced blocks of trials. Observers were instructed to keep their eyes on the central fixation stimulus throughout the trial and to respond by pressing the space bar as quickly as possible when a target was detected. Each trial began with a $100-\mathrm{msec}$ warning tone, a $400-\mathrm{msec}$ pause, and then a cue presented for $100 \mathrm{msec}$. Either 150 or $750 \mathrm{msec}$ after cue onset, the target appeared for $1,500 \mathrm{msec}$ or until a response was made. In each block, the target was omitted on $9 \%$ of trials (catch trials). Trials were separated by a 1,000-msec intertrial interval. Each block of trials was preceded by 10 practice trials. Prior to starting each block, participants were instructed about the probability of a target appearing at a cued location and that cues were nonpredictive.

Standard condition. There were four blocks of trials consisting of displays with two through six peripheral placeholders and a central fixation marker. There were 88 trials in the two-location display $(p=.50), 132$ trials in the three-location display $(p=.33)$, 176 trials in the four-location display $(p=.25)$, and 264 trials in the six-location display $(p=.167)$. Cues and targets were presented in the peripheral placeholders.

Probability-only condition. There were four blocks of trials consisting of displays with two peripheral placeholders and one central placeholder. Peripheral target location probability was .50, $.33, .25$, or .167 . To vary target location probability exclusively, cues were presented peripherally, whereas targets could appear peripherally or centrally. For example, when the peripheral target location probability was .25 (equivalent to the four-location condition in the standard condition), half of all the targets were presented at the central location $(p=.50)$, with the remaining target trials presented peripherally either at a cued location $(p=.25)$ or at an uncued location $(p=.25)$. There were 92 trials with $p=.50,132$ trials with $p=$ $.33,192$ trials with $p=.25$, and 270 trials with $p=.167$.

Location-only condition. There were four blocks of trials consisting of displays with two through six peripheral placeholders (identical to the standard condition) and a central placeholder. To vary number of locations exclusively, cues were presented peripherally while targets could appear anywhere. We constrained the peripheral target location probability to be .167 , regardless of display size. For example, when there were only two peripheral locations, either of which might be cued, a target could be presented peripherally with a probability of $p=.33$ or centrally with a probability of $p=.67$. Thus, the target would appear by chance at either the cued or the uncued location at a probability of $p=.167(p=.33 \div 2$ locations $=.167)$. There were 264 trials in each block.

\section{RESULTS}

Error rates on catch trials ranged from $1.79 \%$ to $5.95 \%$ across conditions, and did not vary as a function of changes in number of locations or target location probability. Errors on all other trials were defined as failures to respond to a target, or responses made less than $200 \mathrm{msec}$ or more than 1,000 msec after target onset. Mean RTs were calculated on nonerror trials separately as a function of CTOA $(150,750)$, target position (cued, uncued), and either number of locations $(2,3,4,6)$, in the standard and locationonly conditions, or peripheral target location probability $(.50, .33, .25, .167)$, in the probability-only condition.

\section{Standard Condition}

At the short CTOA (see top of Table 1), a 2 (target position) $\times 4$ (number of location) within-subjects ANOVA on RTs revealed significant facilitation [main effect of target position: $F(1,34)=5.05, p<.04]$ that did not vary as a function of number of locations [target position $X$ number of locations interaction: $F(3,102)=0.79, p>$ $.50]$. There was, however, a main effect of number of locations on overall RTs $[F(3,102)=4.50, p<.01]$. An identical analysis of errors revealed no significant effects (all $p$ s $>$.07).

At the long CTOA (see bottom of Table 1), the same ANOVA on RTs showed significant $\operatorname{IOR}[F(1,34)=$ $267.59, p<.001]$ that decreased with an increase in number of locations [target position $\times$ number of locations interaction: $F(3,102)=5.16, p<.01]$. A priori polynomial contrasts revealed a significant linear component to this interaction $(p<.001)$, indicating that IOR declined in a linear fashion as the number of locations increased. As at the short CTOA, there was a main effect of number of locations on overall RTs $[F(3,102)=3.24, p<.03]$. An identical analysis of errors revealed no significant effects (all $p \mathrm{~s}>.12)$.

\section{Probability-Only Condition}

At the short CTOA (see top of Table 2), a 2 (target position) $\times 4$ (probability) within-subjects ANOVA revealed a facilitation effect that fell short of significance [main effect of target position: $F(1,35)=2.26, p>.14$ ] and that did not vary as a function of probability [target position $X$ probability interaction: $F(3,105)=0.62, p>.60]$. There was a main effect of probability $[F(3,105)=5.58, p<$ $.01]$ indicating that overall RTs increased with a decrease in probability. An identical analysis of errors revealed no significant effects (all $p \mathrm{~s}>.82$ ). Center RTs at the short CTOA were also analyzed, revealing no effect of probability $(F<1)$.

At the long CTOA (see bottom of Table 2), the same ANOVA on RTs showed significant $\operatorname{IOR}[F(1,35)=$ $63.75, p<.001]$ that did not vary as a function of probabil-

Table 1

Response Times (in Milliseconds) and Cuing Effects for the Standard Condition

\begin{tabular}{|c|c|c|c|c|c|c|c|c|}
\hline \multirow[b]{2}{*}{ Target Position } & \multicolumn{4}{|c|}{ Short CTOA } & \multicolumn{4}{|c|}{ Long CTOA } \\
\hline & 2-Box & 3-Box & 4-Box & 6-Box & 2-Box & 3-Box & 4-Box & 6-Box \\
\hline Cued & 372 & 355 & 362 & 362 & 386 & 371 & 384 & 370 \\
\hline Uncued & 381 & 364 & 375 & 366 & 338 & 333 & 349 & 343 \\
\hline Difference & -9 & -9 & $-13^{*}$ & -4 & $48^{*}$ & $38^{*}$ & $35^{*}$ & $27^{*}$ \\
\hline
\end{tabular}

Note-CTOA, cue-target onset asynchrony. $\quad$ *Indicates a difference significant at $p<.05$. 
Table 2

\begin{tabular}{|c|c|c|c|c|c|c|c|c|}
\hline \multirow[b]{2}{*}{ Target Position } & \multicolumn{4}{|c|}{ Short CTOA } & \multicolumn{4}{|c|}{ Long CTOA } \\
\hline & .50 & .33 & .25 & .17 & .50 & .33 & .25 & .17 \\
\hline Cued & 358 & 365 & 368 & 381 & 355 & 377 & 376 & 387 \\
\hline Uncued & 367 & 371 & 371 & 383 & 335 & 345 & 352 & 355 \\
\hline Center & - & 324 & 323 & 317 & - & 315 & 313 & 307 \\
\hline Cued - uncued & -9 & -6 & -3 & -2 & $20^{*}$ & $32^{*}$ & $24 *$ & $32^{*}$ \\
\hline
\end{tabular}

ity [target position $\times$ probability interaction: $F(3,114)=$ $2.29, p>.08]$. There was also a main effect of probability $[F(3,105)=9.20, p<.001]$ indicating that overall RTs increased with a decrease in probability. An identical analysis on errors revealed no significant effects (all $p \mathrm{~s}>.34$ ). Center RTs at the long CTOA were also analyzed, revealing no effect of probability $[F(2,76)=1.07, p>.10]$.

\section{Location-Only Condition}

At the short CTOA (see top of Table 3), a 2 (target position) $\times 4$ (number of locations) within-subjects ANOVA revealed significant facilitation [main effect of target position: $F(1,33)=7.51, p<.02]$ that did not vary as a function of number of locations [target position $\times$ number of locations interaction: $F(3,199)=1.73, p>.16]$. There was also a main effect of number of locations $[F(3,99)=3.30$, $p<.03]$ indicating that overall RTs decreased as number of locations increased. An identical analysis of error rates revealed no significant effects (all $p \mathrm{~s}>.56$ ). Center RTs at the short CTOA were also analyzed, revealing no effect of number of locations $[F(2,78)=1.70, p>.10]$.

At the long CTOA (see bottom of Table 3), the same ANOVA on RTs showed significant IOR [main effect of target position: $F(1,33)=149.34, p<.001]$ that decreased with an increase in number of locations [target position $\times$ number of locations interaction: $F(3,99)=3.52$, $p<.02$ ]. A priori polynomial contrasts revealed a significant linear component to this interaction $(p<.001)$, indicating that IOR declined in a linear fashion as the number of locations increased. A main effect of number of locations $[F(3,99)=5.31, p<.01]$ showed that overall RTs decreased with an increase in number of locations. An identical analysis of error rates revealed no significant effects (all $p \mathrm{~s}>.05$ ). Center RTs at the long CTOA were also analyzed, revealing no effect of number of locations $[F(2,78)=2.71, p>.05]$.

\section{Summary}

Separate analyses in each condition revealed modest facilitation at the short CTOA that did not vary as a function of variations in probability, number of locations, or both factors combined. Identical analyses at the long CTOA showed strong IOR across conditions that declined with an increase in number of target locations in both the standard and location-only conditions. However, IOR did not vary as a function of changes in probability alone (probability-only condition). Thus, the probability account for changes in IOR with number of target locations is disconfirmed, whereas the capacity-limited and gradient accounts were supported.

To distinguish between these latter two accounts, it is necessary to examine uncued RTs as a function of cuetarget distance. As noted earlier, the capacity-limited account predicts no change in uncued RTs as a function of cue-target distance, and thus no change in IOR as a function of cue-target distance. In contrast, the gradient account predicts that uncued RTs will decrease with an increase in cue-target distance, thereby increasing the IOR effect.

Cue-target distance analysis. Using the procedure outlined in Pratt et al. (1998), we separated uncued RTs at the long CTOA according to distance from the cued location. Thus, depending on the number of target locations, uncued targets could occur at a location opposite the cue $\left(180^{\circ}\right.$ in Figure 1), near-adjacent to the cue $\left(60^{\circ}\right)$, adjacent to the cue $\left(90^{\circ}\right)$, or far-adjacent to the cue $\left(120^{\circ}\right)$. These data can be seen for the standard condition at the top of Table 4, and for the location-only condition at the bottom of Table 4. Examination of these tables shows striking similarities between the two conditions. Confirming this, a 2 (target position) $\times 4$ (number of locations) $\times 2$ (condition: standard, location-only) mixed ANOVA revealed only one interaction, involving condition and number

Table 3

Response Times (in Milliseconds) and Cuing Effects for the Location-Only Condition

\begin{tabular}{|c|c|c|c|c|c|c|c|c|}
\hline \multirow[b]{2}{*}{ Target Position } & \multicolumn{4}{|c|}{ Short CTOA } & \multicolumn{4}{|c|}{ Long CTOA } \\
\hline & 2-Box & 3-Box & 4-Box & 6-Box & 2-Box & 3-Box & 4-Box & 6-Box \\
\hline Cued & 379 & 376 & 371 & 362 & 391 & 390 & 382 & 371 \\
\hline Uncued & 381 & 380 & 380 & 372 & 352 & 349 & 349 & 342 \\
\hline Center & 323 & 324 & 330 & - & 306 & 309 & 315 & - \\
\hline Cued - uncued & -2 & -4 & $-9^{*}$ & $-10^{*}$ & $39^{*}$ & $41^{*}$ & $33^{*}$ & $29^{*}$ \\
\hline
\end{tabular}


Table 4

Uncued Response Times (in Milliseconds) As a Function of Distance

From the Cued Location (Long Cue-Target Onset Asynchrony)

\begin{tabular}{|c|c|c|c|c|c|c|c|c|}
\hline \multirow[b]{2}{*}{ Target Position } & \multicolumn{4}{|c|}{ Response Time } & \multicolumn{4}{|c|}{ Cued - Uncued Difference } \\
\hline & 2-Box & 3-Box & 4-Box & 6-Box & 2-Box & 3-Box & 4-Box & 6-Box \\
\hline \multicolumn{9}{|c|}{ Standard Condition } \\
\hline Opposite $\left(180^{\circ}\right)$ & 338 & & 341 & 335 & $48^{*}$ & & $43^{*}$ & $35^{*}$ \\
\hline Far adjacent $\left(120^{\circ}\right)$ & & 333 & & 337 & & $38^{*}$ & & $33^{*}$ \\
\hline Adjacent $\left(90^{\circ}\right)$ & & & 354 & & & & $30^{*}$ & \\
\hline Near adjacent $\left(60^{\circ}\right)$ & & & & 351 & & & & $19^{*}$ \\
\hline \multicolumn{9}{|c|}{ Location-Only Condition } \\
\hline Opposite $\left(180^{\circ}\right)$ & 352 & & 341 & 330 & $39^{*}$ & & $41^{*}$ & $41^{*}$ \\
\hline Far adjacent $\left(120^{\circ}\right)$ & & 349 & & 338 & & $41^{*}$ & & $33^{*}$ \\
\hline Adjacent $\left(90^{\circ}\right)$ & & & 353 & & & & $29^{*}$ & \\
\hline Near adjacent $\left(60^{\circ}\right)$ & & & & 350 & & & & $21^{*}$ \\
\hline
\end{tabular}

of locations $[F(3,201)=3.74, p<.02]$ and reflecting increasing convergence of overall RTs as the number of locations increased.

Because of these similarities and because the results of the probability-only condition indicated that target location probability did not influence IOR, we combined the data from the standard and location-only conditions in order to increase statistical power (see Table 5). ${ }^{1}$ Examination shows that uncued RTs increased and IOR decreased with an increase in cue-target distance, supporting the gradient account (in particular, see the Average column). Statistical analyses of uncued RTs were conducted on the four- and six-location conditions which included more than one cue-target distance (with four locations, adjacent, opposite; with six locations, near-adjacent, far-adjacent, opposite). Both of these analyses confirmed significant increases in uncued RTs as cue-target distance decreased [four locations, $F(1,68)=24.07, p<.001$; six locations, $F(2,136)=36.89, p<.001]$.

Is there any support for the capacity-limited account in the present data? As mentioned earlier, this account predicts that IOR should decline with an increase in number of target locations irrespective of cue-target distance. We tested this prediction by examining RTs at the opposite location in the two-, four-, and six-location conditions and at the far-adjacent locations in the three- and six-location conditions. In both cases, contrary to the prediction of the capacity-limitation account, pairwise comparisons revealed no significant changes in RTs as a function of display size (all $p \mathrm{~s}>.05)$.

\section{DISCUSSION}

The present study aimed to unravel a paradox in the IOR literature: Although IOR is thought to reflect a mechanism that biases search toward novel locations, overall IOR declines as the number of search locations increases. We tested three possible explanations for this effect: (1) Capacity limitations reduce the availability of resources required for inhibition as display size increases (capacity-limited account); (2) strategic factors sensitive to reductions in target location probability modulate inhibition (probability account); and (3) inhibition declines with distance from the cued location (gradient account). Contrary to the first two accounts, we found that IOR was insensitive to changes in target location probability and changes in number of locations when cue-target distance was controlled. Moreover, we found evidence that inhibi-

Table 5

Combined Data From the Standard and Location-Only Conditions: Uncued Response Times (in Milliseconds) As a Function of Distance From the Cued Location (Long Cue-Target Onset Asynchrony)

\begin{tabular}{|c|c|c|c|c|c|c|c|c|c|}
\hline \multirow[b]{2}{*}{ Target Position } & \multicolumn{4}{|c|}{ Response Time } & \multicolumn{4}{|c|}{ Cued - Uncued Difference } & \multirow[b]{2}{*}{ Average } \\
\hline & 2-Box & 3-Box & 4-Box & 6-Box & 2-Box & 3-Box & 4-Box & 6-Box & \\
\hline \multicolumn{10}{|c|}{ Standard Condition } \\
\hline Cued & 389 & 380 & 383 & 371 & & & & & \\
\hline Uncued & 345 & 341 & 349 & 342 & $44^{*}$ & $39^{*}$ & $34^{*}$ & $29^{*}$ & \\
\hline \multicolumn{10}{|c|}{ Location-Only Condition } \\
\hline Opposite $\left(180^{\circ}\right)$ & 345 & & 341 & 333 & $44^{*}$ & & $42^{*}$ & $38^{*}$ & 42 \\
\hline Far adjacent $\left(120^{\circ}\right)$ & & 341 & & 338 & & $39^{*}$ & & $33^{*}$ & 36 \\
\hline Adjacent $\left(90^{\circ}\right)$ & & & 353 & & & & $30^{*}$ & & 30 \\
\hline Near adjacent $\left(60^{\circ}\right)$ & & & & 350 & & & & $21^{*}$ & 21 \\
\hline
\end{tabular}

Note-Target position (left-hand column) is represented in distance category as well as by angular position around an imaginary circle (see Figure 1). ${ }^{*}$ Indicates a difference significant at $p<.05$. 
tion declined in strength with increasing distance from a cued location. Together, these findings support the gradient account and compel rejection of the probability and capacity-limited accounts.

It should be noted that the inhibitory gradient found here was also obtained by Pratt et al. (1998). However, interpretability of this earlier result was limited in two ways. First, Pratt et al. confounded number of locations with target location probability. Thus, as discussed earlier, it was not possible to distinguish between different theoretical explanations for this finding. Second, while the gradient account was supported by the absence of display-size effects on RTs when cue-target distance was held constant, interpretation of this null effect was compromised by lack of statistical power. In Pratt et al.'s study, 20 participants each viewed 260 trials, which yielded as few as 12 cued trials (six-location condition). In the present study, 105 participants each received a minimum of 44 cued trials per condition. This substantial increase in power increases confidence that our result was not a Type II error.

Why is there a gradient of inhibition? One possibility is that a spatial similarity function underlies the tagging of the cued location with inhibition. In this way, locations that are more similar to the cued location, that is, closer to the cued location, are tagged with more inhibition. This "similarity space" may reflect a coarse resolution of the inhibitory system, which dovetails nicely with evidence for a primitive neural architecture underlying IOR (see, e.g., Sapir, Soroker, Berger, \& Henik, 1999). Another possibility is that this "similarity space" reflects memory confusions between cued and nearby uncued locations. One way to disentangle these possibilities would be to manipulate memory load and the proximity of cued and uncued locations and measure their influences on IOR.

Although evidence for an inhibitory gradient seems incontrovertible, many questions remain about its properties. For instance, is the gradient sensitive to manipulations of object-based memory? Using a multiple-cuing paradigm, Paul and Tipper (2003) found that IOR was stronger and appeared at more of the cued locations when potential target locations were indicated by a salient placeholder, while Birmingham and Pratt (2005) obtained IOR at multiple sequential locations only when potential target locations were indicated by a placeholder. These findings raise the possibility that placeholder objects facilitate more stable memory traces, thereby allowing IOR to be maintained for longer durations, and over greater numbers of cues. Given these temporal effects, the possibility must be considered that the spatial extent of inhibition might also be affected by the presence of placeholders. ${ }^{2}$ Future studies will be required to shed light on this issue.

Finally, we must emphasize that while capacity limitations and top-down control do not explain the effect of number of target locations on IOR, these factors are far from irrelevant. Numerous studies have shown that IOR is sensitive to task difficulty (Castel et al., 2005; Snyder \& Kingstone, in press) and spatial working memory load (Castel et al., 2003) Similarly, studies using the multiple cuing paradigm suggest that IOR is impacted by capacity limitations over time, with IOR occurring at approximately six sequentially cued locations (Snyder \& Kingstone, 2000). Finally, there is evidence that IOR can be adjusted to coincide with when a target is expected to appear (Milliken, Lupiáñez, Roberts, \& Stevanovski, 2003; Mondor, 1999), indicating a sensitivity to top-down modulation. Taken together, these results suggest that IOR is the expression of a complex interplay of numerous mechanisms, including in large part, an inhibitory spatial gradient.

\section{AUTHOR NOTE}

Correspondence relating to this article should be sent to E. Birmingham, Department of Psychology, University of British Columbia, 2136 West Mall, Vancouver, BC, V6T 1Z4 Canada (e-mail: ebirmingham2@, yahoo.ca)

\section{REFERENCES}

Birmingham, E., \& Pratt, J. (2005). Examining inhibition of return with onset and offset cues in the multiple-cuing paradigm. Acta Psychologica, 118, 101-121.

Castel, A. D., Pratt, J., Chasteen, A. L., \& Scialfa, C. T. (2005). Examining task difficulty and the time course of inhibition of return: Detecting perceptually degraded targets. Canadian Journal of Experimental Psychology, 59, 90-98.

Castel, A. D., Pratt, J., \& Craik, F. I. M. (2003). The role of spatial working memory in inhibition of return: Evidence from divided attention tasks. Perception \& Psychophysics, 65, 970-981.

Danziger, S., Kingstone, A., \& SNyder, J. J. (1998). Inhibition of return to successively stimulated locations in a sequential visual search paradigm. Journal of Experimental Psychology: Human Perception \& Performance, 24, 1467-1475.

Gibson, B. S., \& Egeth, H. (1994). Inhibition of return to object-based and environment-based locations. Perception \& Psychophysics, 55, 323-339.

KLEIN, R. [M.] (1988). Inhibitory tagging system facilitates visual search. Nature, 334, 430-431.

KLEIN, R. M., \& MACINNES, W. J. (1999). Inhibition of return is a foraging facilitator in visual search. Psychological Science, 10, 346-352.

Lupiáñez, J., Milliken, B., Solano, C., Weaver, B., \& Tipper, S. P. (2001). On the strategic modulation of the time course of facilitation and inhibition of return. Quarterly Journal of Experimental Psychology, 54A, 753-773.

MAYLOR, E. A. (1985). Facilitatory and inhibitory components of orienting in visual space. In M. I. Posner \& O. S. M. Marin (Eds.), Attention and performance XI (pp. 189-204). Hillsdale, NJ: Erlbaum.

Milliken, B., Lupiáñez, J., Roberts, M., \& STEVAnovski, B. (2003). Orienting in space and time: Joint contributions to exogenous spatial cuing effects. Psychonomic Bulletin \& Review, 10, 877-883.

Mondor, T. A. (1999). Predictability of the cue-target relation and the time-course of auditory inhibition of return. Perception \& Psychophysics, 61, 1501-1509.

PAul, M. A., \& TipPer, S. P. (2003). Object-based representations facilitate memory for inhibitory processes. Experimental Brain Research, 148, 283-289.

Posner, M. I., \& Cohen, Y. (1984). Components of visual orienting. In H. Bouma \& D. G. Bouwhuis (Eds.), Attention and performance $X$ (pp. 531-556). Hillsdale, NJ: Erlbaum.

Posner, M. I., Rafal, R. D., Choate, L. S., \& Vaughan, J. (1985). Inhibition of return: Neural basis and function. Cognitive Neuropsychology, 2, 211-228.

Pratt, J., Adam, J., \& McAuliffe, J. (1998). The spatial relationship between cues and targets mediates inhibition of return. Canadian Journal of Experimental Psychology, 52, 213-216.

Reuter-Lorenz, P. A., Jha, A. P., \& Rosenquist, J. N. (1996). What is inhibited in inhibition of return. Journal of Experimental Psychology: Human Perception \& Performance, 22, 367-378.

Sapir, A., Soroker, N., Berger, A., \& HeniK, A. (1999). Inhibition of return in spatial attention: Direct evidence for collicular generation. Nature Neuroscience, 2, 1053-1054.

SNYDER, J. J., \& Kingstone, A. (2000). Inhibition of return and visual 
search: How many separate loci are inhibited? Perception \& Psychophysics, 62, 452-458.

SNYDER, J. J., \& KingSTONE, A. (in press). Inhibition of return at multiple locations and its impact on visual search. Visual Cognition.

\section{NOTES}

1. Analysis of the combined data produced all of the critical effects from the separated analyses: There were significant main effects of number of locations $[F(3,204)=4.22, p<.01]$ and target position
$[F(1,68)=395.56, p<.001]$ and a number of locations $\times$ target position interaction $[F(3,204)=7.74, p<.001]$. Again, a priori polynomial contrasts revealed a significant linear component to this interaction $(p<$ .001 ), indicating that IOR declined in a linear fashion as the number of locations increased.

2. We thank an anonymous reviewer for bringing up this important issue.

(Manuscript received October 28, 2006; revision accepted for publication January 7,2007 .) 